\title{
Participación educativa en el desarrollo de serious games sobre bullying y uso seguro de Internet: Caminando se hace el camino
}

\section{Educational participation in serious game development about bullying and safe use of the Internet: The path is created by walking}

\author{
Marta Martín del Pozo \\ Universidad de Salamanca \\ mmdp@usal.es \\ Ana García-Valcarcel Muñoz-Repiso \\ Universidad de Salamanca \\ anagv@usal.es \\ Verónica Basilotta Gómez-Pablos \\ Universidad de Salamanca \\ veronicabgp@usal.es
}

Recibido: 02/12/2017

Aceptado: $13 / 12 / 2017$

Publicado: 31/12/2017

\section{RESUMEN}

Los serious games son videojuegos que pueden ser creados con finalidad educativa, siendo fundamental la presencia de profesionales de la educación en su desarrollo. Se presenta en este artículo la participación que por parte de un grupo de profesionales de la educación se está llevando a cabo en el proyecto "eConfidence: Confidence in behaviour changes through serious games" (ref. 732420), financiado por la Unión Europea mediante el programa Horizon 2020 y en el que participan grupos de investigación y empresas. Con este proyecto se busca comprobar una metodología de desarrollo de serious games para promover el cambio de comportamiento en el usuario, probándolo específicamente con el desarrollo de dos serious games, uno sobre uso seguro de Internet y otro sobre bullying, dirigidos a adolescentes. Para su desarrollo, los educadores participan en varias fases, como la revisión de literatura sobre los contenidos de los juegos, definición de métricas educativas o aplicación de prueba piloto en centros escolares. En el texto se presenta un marco teórico sobre ambos constructos (bullying y uso seguro de Internet) y sobre videojuegos y serious games en educación. Posteriormente, se indican los objetivos, las cuestiones metodológicas, los procesos que se están llevando a cabo, los instrumentos, y los participantes y fuentes de información utilizados. Finalmente, se señalan algunos de los resultados generados hasta el momento y conclusiones a las que se ha llegado en base a la situación actual del proyecto, todo ello desde el punto de vista educativo.

\section{PALABRAS CLAVE}

Videojuegos; Serious Games; Bullying; Uso seguro de Internet; Cambio de comportamiento

\section{ABSTRACT}

Serious games are video games that can be designed with an educational purpose, so it is fundamental the presence of professionals from the educational field in their development. This article shows the participation of a group of professionals from the educational field in the project "eConfidence: Confidence in behavioural changes through serious games" (ref. 732420), funded by the European Union through the Horizon 2020 program, in which research groups and companies are taking part. With this project, researchers want to test a serious games development methodology to promote the change of 
behaviour in the users. Also, this methodology is going to be tested with the development of two serious games, one of them about the safe use of the Internet and the other about bullying, targeted for teenagers. For its development, these educators are taking part in several phases, such as literature review about the content of the games, the definition of educational metrics or the implementation of pilot tests in schools. In this text, a theoretical framework on both constructs (bullying and safe use of Internet) is showed and also on video games and serious games in education. Subsequently, we show the educational objectives, the methodological questions and the processes that have been carried out, the instruments, and the participants and sources of information used. Finally, we explain some of the results generated so far and some conclusions that have been reached based on the current situation of the project, all of these from the educational point of view.

\section{KEYWORDS}

Video Games; Serious games; Bullying; Safe use of the Internet; Behaviour change.

\section{CITA RECOMENDADA}

Martínez, M., García-Valcarcel, A. y Basilotta, V. (2017). Participación educativa en el desarrollo de serious games sobre bullying y uso seguro de Internet: caminando se hace el camino. RIITE. Revista Interuniversitaria de Investigación en Tecnología Educativa, 3, 13-24. Doi: http://dx.doi.org/10.6018/riite/2017/312881

\section{Principales aportaciones del artículo y futuras líneas de investigación:}

- Subrayar la importancia de incorporar educadores en el desarrollo de serious games.

- Mostrar las fases en que participan profesionales de la educación en un proyecto interdisciplinario de desarrollo de serious games.

- Mostrar el proceso de investigación cuasiexperimental que permite analizar la efectividad educativa de serious games diseñados para el cambio de comportamientos.

- Aportar información sobre este ámbito a futuros educadores, maestros y pedagogos.

\section{INTRODUCCIÓN}

Los serious games son un tipo de videojuegos con finalidad más allá del entretenimiento, pues en su diseño se ha definido que tuvieran un objetivo educativo, informativo, de entrenamiento o de concienciación social, entre otros. En el caso del desarrollo de serious games con finalidad educativa, se hace necesaria la presencia de especialistas en educación que permita aportar sus conocimientos desde el diseño de los mismos. Como señala Morales (2015), el desarrollo del videojuego no puede hacerse sin una definición clara de los aspectos pedagógicos, por lo que es normal que un equipo de pedagogos ayude a definir los aspectos principales de la agenda educativa del videojuego.

En este artículo se presenta el proyecto "eConfidence: Confidence in behaviour changes through serious games", financiado por la Unión Europea mediante el programa Horizon 2020 Call: H2020-ICT-2016-2017 (Topic: ICT-24-2016) y, en particular, la participación que está realizando en él el grupo GITE-USAL, los procesos llevados a cabo y algunos de los resultados obtenidos hasta el momento desde el punto de vista educativo.

Este proyecto está siendo desarrollado por profesionales de ámbitos del desarrollo informático y tecnológico, game design, educación, psicología y del empresarial, permitiendo promover la sinergia a nivel europeo entre profesionales de diferentes áreas. En particular, los participantes del proyecto son el Instituto Tecnológico de Castilla y León (España), como coordinadores del proyecto, European Schoolnet (Bélgica), Everis (España), Nurogames (Alemania), la Universidad de Salamanca (España) y la Facultad de Humanidades y Ciencias Sociales de la Universidad de Rijeka (Croacia). Se busca comprobar la eficacia de una metodología de desarrollo de serious games que permita promover el cambio de comportamiento en el usuario, probándolo específicamente con el desarrollo de dos juegos, uno sobre uso seguro de Internet y otro sobre bullying. Para ello, ambos juegos serán probados en centros escolares europeos para evaluar los cambios de comportamiento en estudiantes de edades entre 12 y 14 años. Dicho esto, el artículo se divide en diversas secciones que 
permitirán explicar la participación de los profesionales de la educación en este proyecto, así como describir los constructos sobre los cuales se van a desarrollar los serious games y la metodología de investigación utilizada.

\section{ESTADO DEL ARTE}

El bullying y los problemas derivados de un uso no seguro de Internet son dos de las problemáticas a las que se enfrentan los jóvenes en la sociedad actual y ante las cuáles hay que aportar todos los recursos necesarios para evitar los efectos negativos que pueden tener en ellos y ayudarles en un desarrollo personal positivo.

\subsection{Bullying}

Los estudios realizados sobre bullying son muy diversos en cuanto a objetivos, participantes, enfoque de investigación, procedimiento, etc. Considerando la población escolar y siguiendo a Avilés (2006), el bullying se definiría como la intimidación y el maltrato entre alumnado que ocurre de manera repetida y durante el tiempo (es decir, no es un hecho puntual), que se realiza alejado de la presencia y mirada de adultos, con el objetivo de humillar a otra persona y someter abusivamente a dicha víctima indefensa, por parte de un abusón 0 grupo de matones, manifestándose a través de diferentes formas como agresiones físicas, verbales y/o sociales que da lugar a la victimización psicológica y el rechazo grupal en la víctima. En este sentido, se destacan los siguientes indicadores a considerar: repetición, duración, intención, desequilibrio, indefensión, diversidad de formas y consecuencias que genera.

En lo que respecta a los niveles de prevalencia, actualmente, en España, según datos aportados por Sastre (2016) mediante una encuesta realizada a estudiantes entre 12 y 16 años de Educación Secundaria Obligatoria, un 9,3\% indica haber sufrido acoso tradicional en los dos últimos meses, frente a un $6,9 \%$ que considera haber sufrido ciberacoso. Además, un 3,7\% señala haber sufrido ambas modalidades de acoso. En cuanto a las experiencias de acoso vividas, seis de cada diez menores indican que alguien les ha insultado en los últimos meses, reseñando un $22,6 \%$ que esto ha ocurrido frecuentemente.

En cuanto a la prevalencia de la agresión, es decir, los jóvenes que reconocen acosar o ciberacosar a otros, en el estudio de Sastre (2016), un 5,4\% de los encuestados españoles reconoce haber sido responsable de acosar a alguien en los últimos dos meses frente a un $3,3 \%$ que indica haber llevado a cabo ciberacoso hacia otros. Además, un 1,6\% indica haber sido agresor en ambas situaciones.

Sin embargo, este no es un problema únicamente del contexto español, sino que es una problemática a nivel mundial. Por ejemplo, en el estudio de Ditch the Label (2017), en el que participaron estudiantes entre 12 y 20 años de Reino Unido, un 54\% señalaron haber sido acosados en algún momento. Además, entre aquellos que lo habían sufrido, por ejemplo, un $6 \%$ señalaron haberlo sufrido diariamente, un $9 \%$ varias veces a la semana o un $5 \%$ una vez a la semana. En cuanto a las manifestaciones del bullying, considerando aquellos que lo habían sufrido dentro del último año, un 15\% indicaba haber experimentado bullying de tipo verbal constantemente y un $20 \%$ de manera frecuente. En cuanto a bullying de tipo físico, un $5 \%$ señalaba haberlo sufrido constantemente y un $10 \%$ de manera frecuente. Por otro lado, un $12 \%$ manifestaban haber acosado a alguien alguna vez.

Otro ejemplo lo encontramos en Garmy, Vilhjálmsson y Kristjánsdóttir (2017) que describen la frecuencia y variaciones en bullying mediante una muestra representativa nacional de Islandia, con estudiantes de los grados 6, 8 y 10, implicando una media de edades de 11, 13 y 15 años respectivamente. Como instrumento de recogida de datos se utilizó un cuestionario anónimo estandarizado. En cuanto a los resultados, un 7,2\% de los estudiantes de 11 años, un $5,6 \%$ de los de 13 años y un $3,6 \%$ de los de 15 años señalaron ser víctimas al menos 2 o 3 veces por mes. En cuanto a manifestaciones del bullying, por ejemplo, el 6,3\% de los estudiantes de 11 años, el $4,2 \%$ de los de 13 años y el 1,9\% de los de 15 años señalaron haber sido golpeados al menos 2 o 3 veces por mes. O, también, un 4,8\% de los alumnos de 11 
años, un $6.1 \%$ de los estudiantes de 13 años y un $3.5 \%$ de los de 15 años indicaron que se habían burlado de ellos debido al desarrollo corporal.

Foody, Samara y O'Higgins Norman (2017) Ilevaron a cabo un meta-análisis sobre estudios relativos a bullying tradicional y ciberbullying en la República de Irlanda e Irlanda del Norte para conocer la prevalencia entre estudiantes de primaria y secundaria. Se incluyeron 39 estudios, incorporando tanto víctimas como acosadores y aspectos sobre prevalencia, impacto psicológico sobre los menores, políticas y cuestiones sobre intervención. Algunos de los resultados son que la frecuencia de victimización fue de $22.4 \%$ en cuanto a estudiantes de educación primaria y $12,1 \%$ en cuanto a estudiantes de post-primaria, considerando ambos tipos de acoso. Además, en cuanto a la perpetración del bullying, tanto acoso tradicional como online, fue de $9,4 \%$ en primaria y $6.1 \%$ en post-primaria.

\subsection{Uso seguro de Internet}

El uso seguro de Internet hace referencia al uso de manera adecuada y razonable de Internet, conociendo tanto opciones y beneficios que aporta, como riesgos que pueden surgir, consecuencias que pueden tener, medidas a tomar para evitar riesgos, y, en caso de haber ocurrido una situación problemática, cómo afrontarla y qué medidas tomar. El aprendizaje de estas cuestiones se ha vuelto cada vez más necesario, considerando los datos de utilización de Internet y, como señalan Gamito, Aristizabal, Olasolo y Vizcarra (2017), el uso responsable de las TIC y la autonomía digital se han vuelto competencias fundamentales en la sociedad actual. Por ejemplo, en España, según datos del Instituto Nacional de Estadística (2016), la población que ha usado Internet en los últimos tres meses entre 16 y 24 años han sido un 98,6\% de hombres y un $98,2 \%$ de mujeres. En otros países, como por ejemplo en Finlandia, según Statistics Finland (2016) el 100\% de la población entre 16 y 24 años ha usado Internet en los últimos 3 meses y un $97 \%$ usa Internet normalmente varias veces al día. Incluso, en Suecia, según Swedish Media Council (2016), el 65\% de los jóvenes entre 9 y 12 años, el 95\% de los de 13-16 años y de los de 17-18 años, usan Internet todos los días.

Vistos estos datos en cuanto a la expansión de Internet y utilización por los jóvenes, se hace imprescindible adquirir los conocimientos y desarrollar las habilidades y actitudes necesarias que permitan su uso seguro. Más si cabe, considerando los diferentes riesgos que pueden presentarse en su utilización y algunas conductas peligrosas relacionadas con su uso que manifiestan algunos jóvenes. En este sentido, Fernández-Montalvo, Peñalva e Irazabal (2015) analizaron diversas características y el patrón de uso de Internet de 364 estudiantes españoles entre 10 y 13 años, comprobando que entre el uso que realizan de Internet se presentan conductas de riesgo. Entre otras conductas, el $29,9 \%$ da el número de teléfono a alguien, el $13,9 \%$ cuelga datos personales en Internet, el 5,7\% cuelga datos personales de otros, el 5,6\% queda físicamente con alguien desconocido, el 12,8\% añade desconocidos a la lista de amigos, el $12,3 \%$ ha insultado alguna vez por Internet y al $13,7 \%$ le han insultado por Internet.

En este sentido, diversas instituciones y educadores han subrayado la importancia de tomar medidas para usar Internet de manera segura. Por ejemplo, el Cuerpo Nacional de Policía en su página web señala medidas a adoptar por padres o hijos. Algunas son, en cuanto a los padres, que les comenten a sus hijos sobre los peligros del chat y la comunicación online y que revisen los contenidos que pudieran ser perjudiciales para la educación de los hijos. En cuanto a los menores, que no deben dar datos personales si no se está seguro del destinatario o si no son realmente necesarios, ni enviar fotografías personales o de la familia sin que los padres lo autoricen, ni contestar a mensajes extraños o abrir ficheros de los que desconocen su origen. También encontramos la iniciativa PantallasAmigas (http://www.pantallasamigas.net/), que busca el fomento del uso seguro y saludable de las tecnologías y la promoción de la ciudadanía digital responsable en niños y jóvenes. Aporta medidas, consejos e información sobre cuestiones relativas al uso seguro de las tecnologías, como, por ejemplo, sobre identidad digital, privacidad y problemáticas como ciberdelitos, sexting o ciberbullying. 


\subsection{Videojuegos, serious games y educación}

Como se ha visto, tanto la prevención del bullying como el fomento del uso seguro de Internet, se ha vuelto una necesidad imperiosa en nuestra sociedad, para lo cual hay que utilizar todas las herramientas que estén a nuestra disposición, ya sean recursos digitales o no digitales, programas formativos, iniciativas sociales, campañas de concienciación... En este sentido, se está apostando por el uso de un recurso tan extendido como son los videojuegos, de gran interés para nuestros jóvenes y presentes constantemente en sus vidas.

Los videojuegos son uno de los recursos de ocio por excelencia, pero cada vez más se introducen en las aulas y se utilizan con fines educativos. Por ejemplo, aspectos que se han trabajado en diferentes experiencias son: cuestiones de percepción auditiva de tipo musical (García y Raposo, 2013); pensamiento narrativo (Lacasa, 2011); o desarrollo del cálculo mental y resolución de problemas (Capell, Tejada y Bosco, 2017).

Como vemos, los videojuegos se han considerado un formato útil para promover procesos de enseñanza-aprendizaje en torno a diferentes temáticas por sus características interactivas y motivadoras para el alumnado. Sin embargo, se ha ido planteando no solamente la posible utilización educativa de videojuegos creados con fines de entretenimiento o comerciales, sino que se han ido creando propiamente recursos en este formato con objetivos más allá del entretenimiento ya en su propia concepción, entre los que se encuentran objetivos educativos, informativos o, por ejemplo, de entrenamiento. Este tipo de videojuegos se denomina "serious games". Según Michael y Chen (2006) los serious games (o juegos serios) son videojuegos que no tienen como principal fin el entretenimiento o la diversión, sino que tienen otro propósito principal, siendo juegos que utilizan este medio artístico para enviar un mensaje, aportar una experiencia o enseñar una lección.

Centrándonos en la aplicación de este tipo de videojuegos en la educación, también pueden tener diversas finalidades. Por ejemplo, hay experiencias en las que se trabaja la conciencia fonológica y el reconocimiento de palabras en niños disléxicos (Jiménez y Rojas, 2008); la enseñanza de principios de seguridad alimentaria (Rooney, 2011; Rooney y Mac Namee, 2007) o el tratamiento del acoso escolar y la minimización de conductas asociadas (Guerra, 2017), estando esto último en estrecha relación con las temáticas del proyecto eConfidence.

Teniendo en cuenta las posibilidades que ofrecen los serious games, el proyecto eConfidence se ha embarcado en la creación de dos juegos que favorezcan el cambio de comportamiento en cuanto a las dos temáticas señaladas: uso seguro de Internet y prevención del bullying.

\section{MÉTODO}

El proyecto eConfidence, con la participación de los diferentes especialistas, plantea la ejecución de un ciclo de desarrollo considerando 3 etapas: desarrollo de los juegos, testeo/recolección de datos y análisis de los datos. En este sentido, aunque el proyecto tiene diversos objetivos, de carácter científico, tecnológico, social y económico, nos centramos en los aspectos educativos, y en la participación de los profesionales de la educación en este proyecto.

Así pues, desde el ámbito educativo, los objetivos a alcanzar con este proyecto son:

- Probar la eficacia del producto (serious games), en relación al cambio de comportamiento de los estudiantes en cuanto a uso seguro de Internet y bullying (en función de cada juego).

- Incrementar conocimientos, mejorar actitudes y cambiar comportamientos de los estudiantes en relación al uso seguro de Internet.

- Incrementar conocimientos, mejorar actitudes y cambiar comportamientos de los estudiantes en cuanto a las situaciones de bullying, los efectos negativos y la protección de la víctima. 
Además, con ello se busca incrementar la aceptación de los serious games como herramienta educativa en las escuelas participantes y como herramientas que permiten el cambio de comportamiento en entornos formativos como escuelas, empresas, centros terapéuticos...

Considerando lo anterior, las tareas asumidas por los profesionales de la educación en el proyecto eConfidence en relación a su contribución al diseño y desarrollo de los serious games, son las siguientes:

- Revisión del estado de la cuestión sobre uso seguro de Internet y situaciones de bullying en relación a los jóvenes.

- Revisión del estado de la cuestión sobre posibilidades educativas de los videojuegos para adolescentes de 12 a 16 años.

- Elaboración de instrumentos de recogida de información que es preciso construir ad hoc para el estudio.

- Propuestas de procesos metodológicos para la creación de serious games desde el punto de vista pedagógico para conseguir cambios de comportamiento.

- Definición de métricas educativas para los videojuegos con objeto de analizar la evolución de los estudiantes en la prueba piloto.

- Aplicación de prueba piloto en centros escolares, incluyendo la realización de pretest, tratamiento (uso de juegos) y postest.

- Análisis de los datos de la prueba piloto.

En cuanto a los diseños metodológicos para su realización, por una parte, las etapas de revisión y conceptualización se plantean a modo de revisión sistemática de literatura, permitiendo la recogida y análisis de datos de estudios previos y el establecimiento de una base sólida para los futuros pasos. Por otro lado, en cuanto al estudio piloto, se planifica a través de una metodología cuasiexperimental con un diseño pretest-postest con grupo experimental y grupo control. El grupo experimental recibirá un tratamiento consistente en la utilización de los videojuegos, mientras que el grupo de control no recibirá dicho tratamiento. Ambos grupos responderán los test diseñados para la recogida de información al inicio y al final del proceso.

\subsection{Instrumentos de recogida de información}

Las revisiones de literatura se trabajan a través de fichas y hojas de datos que permiten recoger los aspectos fundamentales de cada uno de los artículos para su posterior análisis.

En cuanto a la prueba piloto, algunas de las variables que se quieren analizar son: conocimiento y actitud hacia el uso seguro de Internet, conocimiento sobre bullying, asertividad o habilidades sociales. Algunos de los instrumentos a utilizar son algunos ítems del Children's Assertive Behavior Scale (Michelson y Wood, 1982), una parte de la subescala sobre habilidades sociales del Matson Evaluation of Social Skills with Youngsters (Matson, Rotatori, y Helsel, 1983) y otros instrumentos elaborados ad hoc, como un cuestionario de elección múltiple que presenta situaciones ficcionales relacionadas con el uso seguro de Internet. Para más información, en el siguiente enlace se encuentra un dosier sobre los instrumentos para la prueba

piloto: http://www.econfidence.eu/documents/407765/497360/eConfidence MeasurementInstruments PilotTest summary.pdf/b128849d-afc5-43fd-b3c3-e51495322eef.

\subsection{Fuentes de información y participantes}

En las revisiones de literatura se consideran aquellas publicaciones de calidad que aparecen en bases de datos como ScienceDirect, SpringerLink, Dialnet y Emerald Insight. Se utilizan las funciones adecuadas para permitir una búsqueda efectiva y usando los términos clave correspondientes.

En cuanto al estudio piloto, los participantes son escolares entre 12 y 14 años que estudian en 10 centros educativos (españoles y de países de habla inglesa) en el año académico 2017-2018. La muestra se selecciona mediante muestreo por conveniencia, para lo que se abre una convocatoria pública que permite a los centros presentarse como candidatos 
al proyecto. Una vez finalizado el proceso de candidaturas, se seleccionan diez centros mediante entrevistas online en base a criterios sobre infraestructura tecnológica, disponibilidad de alumnado o experiencia previa en proyectos europeos, entre otros.

\section{RESULTADOS}

Procedemos a indicar los resultados obtenidos hasta el momento actual en relación a las fases señaladas.

\subsection{Revisión del estado de la cuestión sobre uso seguro de Internet y situaciones de bullying en relación a los jóvenes}

Se revisaron estos constructos, analizando un gran número de documentos científicos, alcanzando una base sólida de conocimientos respecto a los conceptos más relevantes. En cuanto al trabajo del grupo de profesionales de educación, se revisó la documentación sobre uso seguro de Internet. El trabajo realizado se plasmó en un documento (Deliverable compartido con los socios del consorcio) sobre aspectos educativos, en forma de guía con orientaciones, medidas y cuestiones a considerar para la promoción del uso seguro de Internet. La guía está estructurada en siete partes relativas a seguridad de los dispositivos, registro online y contraseñas, navegadores, email, redes sociales, mensajería instantánea y gestión de las imágenes. En cada apartado se señalan indicadores de un comportamiento seguro sobre dicho aspecto. Por ejemplo, en cuanto a registro online y contraseñas, quién debe saber las contraseñas, la lectura de los términos de uso de las aplicaciones o características para contraseñas fuertes. Esta guía ha sido utilizada como base para la elaboración del videojuego sobre esta temática por la empresa encargada de esta tarea.

\subsection{Revisión del estado de la cuestión sobre posibilidades educativas de los videojuegos para adolescentes de 12 a 16 años}

Se realizó una revisión sistemática de literatura sobre el uso de videojuegos en educación secundaria para conocer los resultados de su implementación en este nivel. Se localizaron 951 artículos utilizando los siguientes términos: games and "secondary education" and learning. Se aceptaron 23 considerando criterios de inclusión y exclusión. Algunos de los resultados es que la mayoría de los juegos utilizados eran juegos diseñados para el aprendizaje (es decir, serious games) frente a videojuegos de entretenimiento/comerciales, siendo los tipos más usados tutoriales animados, juegos de simulación y puzle. Además, la mayoría de los estudios indicaban que utilizando videojuegos (sin diferenciar entre serious games y videojuegos de entretenimiento) se adquirían conocimientos y se mejoraba la comprensión del contenido, y también otros indicaban que mejoraba la motivación y tenía efectos emocionales.

\subsection{Elaboración de instrumentos de recogida de información que es preciso construir ad hoc para el estudio}

Ha sido precisa la elaboración de algunos de los instrumentos de recogida de información. En nuestro caso, se ha diseñado un cuestionario que permitiera evaluar el conocimiento, comportamiento y actitudes sobre uso seguro de Internet. Se trata de un cuestionario de elección múltiple que presenta situaciones en las que se pueden encontrar los jóvenes relacionadas con uso de Internet.

\subsection{Propuestas de procesos metodológicos para la creación de serious games desde el punto de vista pedagógico para conseguir cambios de comportamiento}

Se revisó literatura sobre el proceso de diseño y creación de serious games y elementos a tener en cuenta desde un punto de vista pedagógico, considerando, entre otros, las aportaciones de Carvalho et al. (2015), De la Hera (2013) y Rooney (2012). Después de la 
revisión, como resultado de este proceso se plantearon modelos y procesos metodológicos para la creación de los serious games del proyecto desde un punto de vista educativo.

\subsection{Definición de métricas educativas para los videojuegos con objeto de analizar la evolución de los estudiantes en la prueba piloto}

Se definieron conceptualmente las métricas de los videojuegos, las cuales permiten la recogida de datos mientras el jugador/estudiante está jugando, permitiendo analizar la evolución de estos a lo largo de la prueba piloto y aportar información complementaria a la del pretest y postest. Una vez definidas, el equipo desarrollador puede implementarlas en los videojuegos. Algunas de las métricas que se han planteado y que forman parte de los resultados de este proceso son, por ejemplo, las opciones de diálogo escogidas por los jugadores durante su participación en el juego, considerando conjuntamente este dato con otros datos como: ID del jugador/estudiante, marca temporal de selección de dicha opción de diálogo, género del jugador en la vida real y en el juego, tiempo de reacción entre la aparición de opciones de diálogo y la elección de una, y puntos en diversas habilidades. A modo de explicación, esto nos permitirá, por ejemplo, conocer la evolución de los sujetos en cuanto a su elección de opciones más o menos adecuadas, pudiendo ocurrir, por ejemplo, en el juego sobre bullying, que algunos estudiantes escojan opciones más asertivas antes que otros.

\subsection{Aplicación de la prueba piloto en centros escolares, incluyendo la realización de pretest, tratamiento y postest}

Actualmente el proyecto se encuentra en la fase de desarrollo del estudio piloto. Se comenzará a aplicar los pretest en los centros en noviembre de 2017. La aplicación de los serious games, que supone el juego de los niños del grupo experimental durante aproximadamente 6 horas en los centros, se realizará en marzo de 2018, bajo la supervisión de un coordinador en cada centro. Posteriormente, una vez finalizada la fase de juego (sólo grupo experimental), se aplicará el pretest con objeto de analizar el cambio de comportamiento generado en los estudiantes, en comparación con el grupo control.

\subsection{Análisis de los datos de la prueba piloto}

El análisis de los datos del estudio piloto se realizará en fechas posteriores a la aplicación del postest (en mayo y junio de 2018), por lo que actualmente no disponemos de datos. En esta fase podremos responder si los videojuegos han sido efectivos para conseguir aquello que se proponían, es decir, si han logrado cambiar el comportamiento de los estudiantes en cuanto al uso seguro de Internet y las situaciones de bullying. De este modo, los juegos quedarán validados como recursos educativos capaces de generar el cambio de comportamiento, y así mismo, se validará la metodología empleada para el diseño de estos serious games.

\section{DISCUSIÓN Y CONCLUSIONES}

El proceso de desarrollo de un serious game es un proceso arduo, tanto desde un punto de vista educativo como técnico (informático), pero que a través de la sinergia de los diferentes profesionales implicados en un proyecto como eConfidence puede generar no sólo juegos efectivos sino conocimiento para el diseño de otros videojuegos. En este camino, se hace relevante la presencia de educadores y pedagogos que contribuyan a clarificar los aspectos relativos al ámbito educativo.

En este artículo, hemos visto las fases en las que ha participado un grupo de profesionales de la educación que colabora en un proyecto de desarrollo de una metodología para el diseño de videojuegos capaces de cambiar los comportamientos, y específicamente en el desarrollo de dos serious games, uno sobre bullying y otro sobre uso seguro de Internet. Estas dos temáticas hacen muy relevante la presencia de especialistas de la educación por ser problemáticas ligadas a dicho ámbito. El bullying es sufrido por parte de la población escolar en diferentes formas (Ditch the Label, 2017; Garmy, Vilhjálmsson y Kristjánsdóttir, 2017; Foody, Samara y O'Higgins Norman, 2017; Sastre, 2016), siendo un problema que no se limita a un país en particular, sino que ocurre a nivel internacional. Por otro lado, la mayor parte de los jóvenes usan Internet (Instituto Nacional de Estadística, 2016; Statistics Finland, 2016; Swedish 
Media Council 2016), pero en algunas ocasiones presentan conductas de riesgo como colgar datos personales en Internet, quedar físicamente con alguien desconocido o insultar o ser insultado por Internet (Fernández-Montalvo, Peñalva e Irazabal, 2015), por lo que en la sociedad actual el uso responsable de las TIC y la autonomía digital se han vuelto competencias fundamentales (Gamito, Aristizabal, Olasolo y Vizcarra, 2017) para evitar estos riesgos.

Teniendo presente que es preciso aportar todos los recursos necesarios para solucionar estas problemáticas y que los videojuegos se han mostrado como una herramienta que, de manera atractiva y lúdica, pueden transmitir información, contribuir a desarrollar habilidades, promover emociones, cambiar actitudes y comportamientos (como se ejemplifica en Capell, Tejada y Bosco, 2017; García y Raposo, 2013; Guerra, 2017; Guerra y Revuelta, 2015; Jiménez y Rojas, 2008; Lacasa, 2011; Rooney, 2011; Rooney y Mac Namee, 2007; y también en los datos obtenidos en la revisión de literatura realizada en una de las fases), se está llevando a cabo un proyecto en el que, para probar una nueva metodología de desarrollo de serious games, se desarrollan dos juegos, cada uno dedicado a una de estas temáticas. Para su desarrollo, se ha contado con un grupo de profesionales de la educación que ha colaborado en las tareas que requiere el desarrollo de los juegos, asesorando sobre aspectos pedagógicos, haciendo realidad lo que indica Morales (2015), que el desarrollo de un videojuego serio no puede hacerse sin una definición clara de los aspectos pedagógicos, remarcando la importancia de la participación de un equipo de pedagogos o educadores.

En cuanto a limitaciones, señalamos la necesidad de mayor formación sobre el ámbito de los serious games por parte de los profesionales de la educación, con objeto de poder participar en procesos de creación, uniéndose a equipos interdisciplinares para este fin. De este modo, como prospectiva, esperamos poder aportar más formación a nuestros titulados del ámbito de la educación, mediante la incorporación de estos temas en asignaturas relacionadas con la integración de las TIC en la educación, así como mediante la organización de actividades formativas.

Para finalizar, vista la importancia de la presencia de este tipo de profesionales en el desarrollo de serious games y la expansión de este tipo de recursos, subrayamos la necesidad de incrementar la formación de los educadores y pedagogos sobre el diseño e integración de estos recursos en el aula (Martín, García-Valcárcel y Hernández, 2016). Esto permitiría un mejor conocimiento sobre el tema y aportaciones a este campo que son demandadas desde otros ámbitos profesionales, así como abriría más puertas profesionales de futuro a nuestros egresados de las titulaciones de educación.

\section{ENLACES}

eConfidence (2017) D.2.1. Information dossier on researched topics and indicators to assess educational possibility of the video games. Recuperado de http://www.econfidence.eu/documents/407765/497360/eConfidence InformationdossieronRese archedTopics summary.pdf/7988d4f3-1641-4494-969b-8fd293061539

eConfidence (2017) D.2.2. Information dossier on measurement instruments for the pilot
test. http://www.econfidence.eu/documents/407765/497360/eConfidence MeasurementInstruments PilotTest summary.pdf/b128849d-afc5-43fd-b3c3-e51495322eef

eConfidence (2017) Educational benefits of eConfidence serious games. Recuperado de http://www.econfidence.eu/documents/407765/497360/eConfidence EducationalBenefitsSeriou sGames July2017.pdf/5669dbeb-8171-404b-810c-5be16a742671

Página web del proyecto eConfidence (2017). Recuperado de http://www.econfidence.eu

\section{RECONOCIMIENTOS}


Este proyecto ha recibido financiación del Programa de Investigación e Innovación de la Unión Europea Horizon 2020 bajo el Grant Agreement No 732420.

Esta publicación refleja solamente el punto de vista de los autores. En este sentido, no representa el punto de vista de la Comisión Europea y la Comisión Europea no es responsable por el uso que pueda realizarse de la información que contiene. Además, en el caso del primer autor, agradecemos la financiación recibida de una ayuda predoctoral del Programa FPU (Formación del Profesorado Universitario) del Ministerio de Educación, Cultura y Deporte. En cuanto al tercer autor, agradecemos la financiación recibida de una ayuda predoctoral de la Junta de Castilla y León, cofinanciada por el Fondo Social Europeo.

\section{REFERENCIAS BIBLIOGRÁFICAS}

Avilés, J. M. (2006). Bullying: el maltrato entre iguales. Agresores, víctimas y testigos en la escuela. Salamanca: Amarú.

Capell, N., Tejada, J. y Bosco, A. (2017). Los videojuegos como medio de aprendizaje: un estudio de caso en matemáticas en Educación Primaria. Píxel-Bit. Revista de Medios y Educación, 51, 133-150. doi: 10.12795/pixelbit.2017.i51.09

Carvalho, M., Bellotti, F. Berta, R., De Gloria, A., Islas-Sedano, C., Baalsrud, J., Hu, J. y Rauterberg, M. (2015). An activity theory-based model for serious games analysis and conceptual design. Computers \& Education, 87, 166-181. doi: 10.1016/j.compedu.2015.03.023

Cuerpo Nacional de Policía (s.f.). Recuperado de www.policia.es

De la Hera, T. (2013, Agosto). A conceptual model for the study of persuasive games. Proceedings of DiGRA 2013: DeFragging Game Studies, Atlanta, EEUU. Recuperado de http://www.digra.org/wp-content/uploads/digital-library/paper 13.pdf

Ditch the Label (2017). The annual bullying survey 2017. Recuperado de https://www.ditchthelabel.org/wp-content/uploads/2017/07/The-Annual-Bullying-Survey2017-1.pdf

Fernández-Montalvo, J., Peñalva, M. A. e Irazabal, I. (2015). Hábitos de uso y conductas de riesgo en Internet en la preadolescencia. Comunicar, 44, 113-120. doi: 10.3916/C44-201512

Foody, M., Samara, M. y O'Higgins Norman, J. (2017). Bullying and cyberbullying studies in the school-aged population on the island of Ireland: A meta-analysis. British Journal of Educational Psychology, 87(4), 535-557. doi: 10.1111/bjep.12163

Gamito, R., Aristizabal, P., Olasolo, M. y Vizcarra, M. T. (2017) La necesidad de trabajar los riesgos de Internet en el aula. Profesorado, Revista de Currículum y Formación del Profesorado, 21(3), 409-426.

García, M. F. y Raposo, M. (2013) Trabajando con videojuegos en el aula: una experiencia con Wii Music. Tendencias Pedagógicas, 22, 45-58.

Garmy, P., Vilhjálmsson, R. y Kristjánsdóttir, G. (2017). Bullying in School-aged Children in Iceland: A Cross-sectional Study. Journal of Pediatric Nursing. doi: 10.1016/j.pedn.2017.05.009

Guerra, J. (2017). Estudio evaluativo de prevención del acoso escolar con un videojuego (Tesis doctoral). Universidad de Extremadura. Recuperado de http://dehesa.unex.es/bitstream/handle/10662/6030/TDUEX 2017 Guerra Antequera.pdf?s equence $=1$ \&isAllowed $=y$ 
Guerra, J. y Revuelta, F. I. (2015). Videojuegos precursores de emociones positivas: propuesta metodológica con Minecraft en el aula hospitalaria. IJERI International Journal of Educational Research and Innovation, 3, 105-120.

Instituto Nacional de Estadística (2016). Encuesta sobre Equipamiento y Uso de Tecnologías de la Información y Comunicación en los Hogares. Población que usa Internet (en los últimos tres meses). Recuperado de http://www.ine.es/ss/Satellite? L=es ES\&c=INESeccion C\&cid=1259925528782\&p=1254735 110672\&pagename $=$ ProductosYServicios\%2FPYSLayout

Jiménez, J. E. y Rojas, E. (2008). Efectos del videojuego Tradislexia en la conciencia fonológica y reconocimiento de palabras en niños disléxicos. Psicothema, 20(3), 347-353.

Lacasa, P. (2011). Los videojuegos. Aprender en mundos reales y virtuales. Madrid: Morata.

Martín, M., García-Valcárcel, A. y Hernández, A. (2016). Video games in teacher: design, implementation and assessment of an educational proposal. En F. J. García- Peñalvo (2016) Proceedings TEEM'16. Fourth International Conference on Technological Ecosystems for Enhancing Multiculturality (pp.1147-1154). New York: ACM.

Matson, J. L., Rotatori, A. F. y Hensel, W. J. (1983) Development of a rating scale to measure social skills in children: the Matson Evaluation of Social Skills with Youngsters (MESSY). Behaviour research and therapy, 21(4), 335-340.

Michael, D. y Chen, S. (2006). Serious Games: Games that Educate, Train and Inform. Boston: Thomson Course Technology.

Michelson, L. y Wood, R. (1982). Development and psychometric properties of the Children's Assertive Behavior Scale. Journal of Behavioral Assessment, 4(1), 3-13. doi: 10.1007/BF01321378

Morales, J. (2015). Serious games: diseño de videojuegos con una agenda educativa y social. Barcelona: UOC

Página oficial de la DGP (s.f.). Consejos al Ciudadano. Recuperado de https://www.policia.es/consejos/internet.html

PantallasAmigas: Por un uso seguro y saludable de Internet, la telefonía móvil y los videojuegos - Por una ciudadanía digital responsable (2004-2017). Recuperado de http://www.pantallasamigas.net/

Rooney, P. (2011). Harnessing serious games in higher education: a case study (Tesis Doctoral). Universidad de Sheffield.

Rooney, P. (2012). A Theoretical Framework for Serious Game Design: Exploring Pedagogy, Play and Fidelity and their Implications for the Design Process. International Journal of Game-based Learning, 2(4), 41-60. doi: 10.4018/ijgbl.2012100103

Rooney, P. y Mac Namee, B. (2007, marzo). Students @ play: serious games for learning in higher education. Presentado en INTED2007, 1st International Technology, Education and Development Conference, Valencia. Recuperado de https://arrow.dit.ie/scschcomcon/28/

Sastre, A. (Coord.) (2016) Yo a eso no juego. Bullying y ciberbullying en la infancia. Madrid: SAVE THE CHILDREN España. Recuperado de https://www.savethechildren.es/sites/default/files/imce/docs/yo a eso no juego.pdf

Statistics Finland (2016) Official Statistics of Finland (OSF): Use of information and communications technology by individuals. Helsinki: Statistics Finland. Recuperado de http://www.stat.fi/til/sutivi/2016/sutivi 2016 2016-12-09 tie 001 en.html 
Swedish Media Council (2016). Ungar och medier 2015. Recuperado de http://statensmedierad.se/download/18.7a953dba14fef1148cf3b32/1442841939189/Ungaroch-medier-2015.pdf

\title{
INFORMACIÓN SOBRE LAS AUTORAS
}

\author{
Marta Martínez del Pozo \\ Universidad de Salamanca
}

Personal Investigador en Formación con una ayuda predoctoral FPU (Formación del Profesorado Universitario) del Ministerio de Educación, Cultura y Deporte, en el Departamento de Didáctica, Organización y Métodos de Investigación de la Universidad de Salamanca. Miembro del Grupo de Investigación GITE-USAL. Licenciada en Pedagogía y Máster TIC en Educación: análisis y diseño de procesos, recursos y prácticas formativas por la Universidad de Salamanca. Realizando tesis doctoral sobre videojuegos, aprendizaje colaborativo y formación docente. Sus líneas de investigación son: uso de las TIC en educación, videojuegos como herramienta de aprendizaje, serious games, game-based learning y formación docente.

Grupo de investigación: http://gite213.usal.es/

ResearchGate: https://www.researchgate.net/profile/Marta Martin Del Pozo

Google Scholar: https://scholar.google.es/citations?user=a6czYmMAAAAJ\&hl=es

Academia.edu: http://usal.academia.edu/MartaMartin

Linkedln: https://es.linkedin.com/in/marta-mart\%C3\%ADn-del-pozo-698b6236

Blog: http://videojuegosmasaprendizaje.blogspot.com.es/

\section{Ana García-Valcarcel Muñoz-Repiso \\ Universidad de Salamanca}

Licenciada y doctora en Ciencias de la Educación. Catedrática de Tecnología Educativa en la Facultad de Educación de la Universidad de Salamanca. Directora del Grupo de Investigación GITE-USAL, Grupo de Excelencia de la Junta de Castilla y León y Directora del Máster Oficial Las TIC en Educación: análisis y diseño de procesos, recursos y prácticas formativas de la Universidad de Salamanca. Sus líneas de investigación son: tecnología educativa, integración de las TIC en los procesos de enseñanzaaprendizaje, evaluación de programas y proyectos educativos de innovación, formación del profesorado.

Grupo de investigación: http://gite213.usal.es/

Web personal: http://diarium.usal.es/anagv/informacion-3/

ResearchGate: https://www.researchgate.net/profile/Ana Garcia-Valcarcel

Google Scholar: https://scholar.google.es/citations?user=loXQH54AAAAJ\&hl=es

Linkedln:https://es.linkedin.com/in/ana-garcia-valc\%C3\%A1rcel-mu\%C3\%B1oz-repiso-76a7a032

\section{Verónica Basilotta Gómez-Pablos \\ Universidad de Salamanca}

Personal Investigador en Formación en el Departamento de Didáctica, Organización y Métodos de Investigación de la Universidad de Salamanca, con una ayuda predoctoral de la Junta de Castilla y León, cofinanciada por el Fondo Social Europeo. Miembro del Grupo de Investigación GITE-USAL. Licenciada en Psicopedagogía y Máster TIC en Educación: análisis y diseño de procesos, recursos y prácticas formativas por la Universidad de Salamanca. Sus líneas de investigación son: el aprendizaje basado en proyectos, el aprendizaje colaborativo y la integración de la tecnología en los procesos de aprendizaje.

Grupo de investigación: http://gite213.usal.es/

ResearchGate: https://www.researchgate.net/profile/Veronica Basilotta Gomez-Pablos

Google Scholar: http://scholar.google.es/citations?user=q-ReVQ8AAAAJ\&hl=es

Linkedln: https://es.linkedin.com/in/veronicabasilotta

\section{$(\mathrm{oc}) \mathbf{B Y}-\mathrm{NC}$}

Los textos publicados en esta revista están sujetos a una licencia de Reconocimiento 4.0 España de Creative Commons. Puede copiarlos, distribuirlos, comunicarlos públicamente y hacer obras derivadas siempre que reconozca los créditos de las obras (autoría, nombre de la revista, institución editora) de la manera especificada por los autores o por la revista. La licencia completa se puede consultar en:Licencia Creative Commons Atribución-NoComercial-Compartir por igual 4.0 Internacional 